\title{
Research on Architecture for B2B E-commerce Platform
}

\author{
Lijun Wang ${ }^{1, a}$, Linchuang Zhang ${ }^{2, b}$ \\ ${ }^{1}$ Basic Teaching and Research Institute, Bohai University, Jinzhou, 121013, China \\ ${ }^{2}$ College of Information Science and Technology, Bohai University, Jinzhou, 121013, China \\ ajsma1111@163.com, b761408020@qq.com
}

Keywords: B2B; E-commerce platform; architecture; processing flow; system function; technology framework

\begin{abstract}
B2B refers to Business to Business, that is, between enterprises and enterprises through the Internet for products, services and information exchange, but also for the strategic cooperation between enterprises to provide a basis. In order to improve the efficiency and quality of B2B e-commerce platform development, this paper studies the architecture. First, based on B2B e-commerce transaction process, designed processing flow architecture; then, designed by the "Business advertising, Merchandise display, Member center, Shopping system, Marketing management, Search this site, News system and Help center" and so on Finally, we design a technology framework architecture composed of "Presentation layer, Control layer, Business logic layer and Data persistence layer".
\end{abstract}

\section{Introduction}

E-commerce refers to commercial trade in a wide range of activities on the Internet, open network environment, based on the Browser/Server application, the buyers and sellers do not meet business activities, realize online shopping, online transactions and online merchant electronic payment of a new business model and a variety of business activities, business activities and financial activities and the related activities of the integrated services. The electronic commerce includes the following meanings: e-commerce is a business, the use of advanced information technology; electronic commerce makes a virtual market place to exchange; e-commerce "is a collection of modern information technology" and "business"; electronic commerce is a kind of idea, rather than simply using electronic facilities to complete business activities.

E-commerce refers to the wide range of commercial trade activities in the Internet open environment, based on Browser / Server application, buyers and sellers do not meet the business activities to achieve online shopping, online transactions between merchants and online electronic payment, As well as a variety of business activities, trading activities, financial activities and related integrated service activities of a new business model. E-commerce contains the following meaning: e-commerce is a way of using advanced information technology trading; e-commerce created a virtual market exchange places; e-commerce is "modern information technology" and "business" collection; e-commerce is a Concept, rather than simply using electronic facilities to complete business activities.

B2B to make transactions between enterprises to reduce the number of businesslike work processes and management costs, the specific advantages are reflected in four aspects: First, reduce procurement costs. Enterprises through the establishment of inter-enterprise e-commerce with suppliers to achieve online automatic procurement, can reduce the two sides for the transaction of human, material and financial resources. Second, reduce inventory costs. Enterprises through the upstream and downstream suppliers and downstream customers to establish e-commerce system between enterprises to achieve sales to produce, to set production for the efficient operation of logistics and unity, the maximum control of inventory. Third, save the turnaround time. Enterprises and suppliers and customers through the establishment of a unified e-commerce system to achieve the enterprise's suppliers and business customers to communicate and trade directly to reduce 
turnover. Fourth, expand market opportunities. Enterprises through the potential customers with the establishment of online business relations, covering the original difficult to cover the traditional channels of the market, increase the market opportunities for enterprises.

The software architecture is a structured element, that is, a set of components, including processing components, data structures, and connection components. The processing component is responsible for processing the data, the data component is the processed information, and the connection component connects the different parts of the architecture. The scale of today's software systems is getting bigger and bigger, and the structure is becoming more and more complex. This paper is based on B2B e - commerce platform, after the description, unified, abstract, generalized and instantiated processing, summed up the architecture, software reuse to provide strong support for improving the system to facilitate.

\section{Processing Flow Architecture}

The B2B electronic commerce transaction process is divided into 8 steps: step first, business customers to vendors ordering, first issued a "customer order", including the product name and quantity of information; the second step, sellers receive "user orders", query products to suppliers according to user requirements, the issue of "order" order "; the third step, the supplier received and verified after the" order "after the return to the vendor" order "answer; the fourth step, to meet the seller to confirm supplier business customers" user orders "requirements, to transport operators issued relevant goods" transportation query "; the fifth step, transport operators on receipt of the" transport inquiry ", to return to the seller transport inquiry answer; the sixth step in the confirmation of transportation is no problem, immediately to the seller's Customer's "customer order", a satisfactory answer, at the same time, to the supplier issued a "Notice of delivery", and notify the carrier transport; the seventh step, the carrier received the "transport notice" after the start of delivery. The business customer then issues a payment notice to the payment gateway". Payment gateway and bank settlement notes, etc. eighth step, payment gateway to the seller issued a successful transaction "transfer notice." The architecture of the processing flow designed according to the above transaction process is shown in Fig. 1.

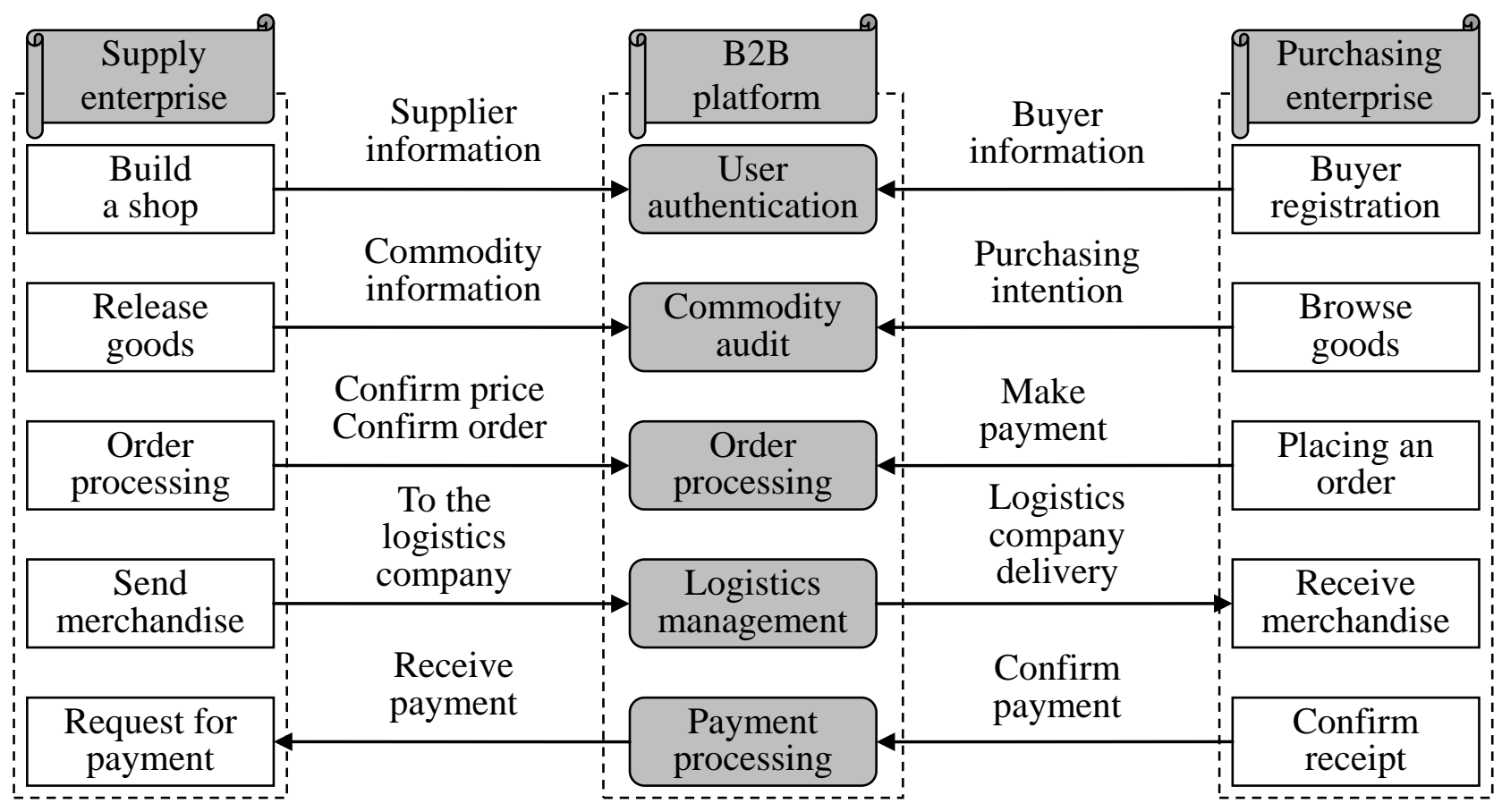

Fig. 1. Processing flow architecture on B2B E-commerce platform 


\section{System Function Architecture}

The functional architecture is the result of the simulation, which decomposes the functions of the system and represents the graphs in terms of functional dependencies. Each rectangle in the diagram is called a functional module. Each subsystem of the management information system can be regarded as the function of the lower layer of the system target, and each of the functions can continue to be decomposed into more functions. Function decomposition process from large to small, from coarse to fine, from top to bottom. Conceptually, the upper layer functions to control the underlying functions, the more general the upper functions, the lower the more specific functions. The process of functional decomposition is a process from abstract to concrete, from complex to simple. The function module according to the specific circumstances of larger or smaller, the decomposition of function modules can be minimum each process in a program, and the larger function module can be a set of program to complete a task. Combined with previous research literature, the system function architecture of this paper is shown in Fig. 2.

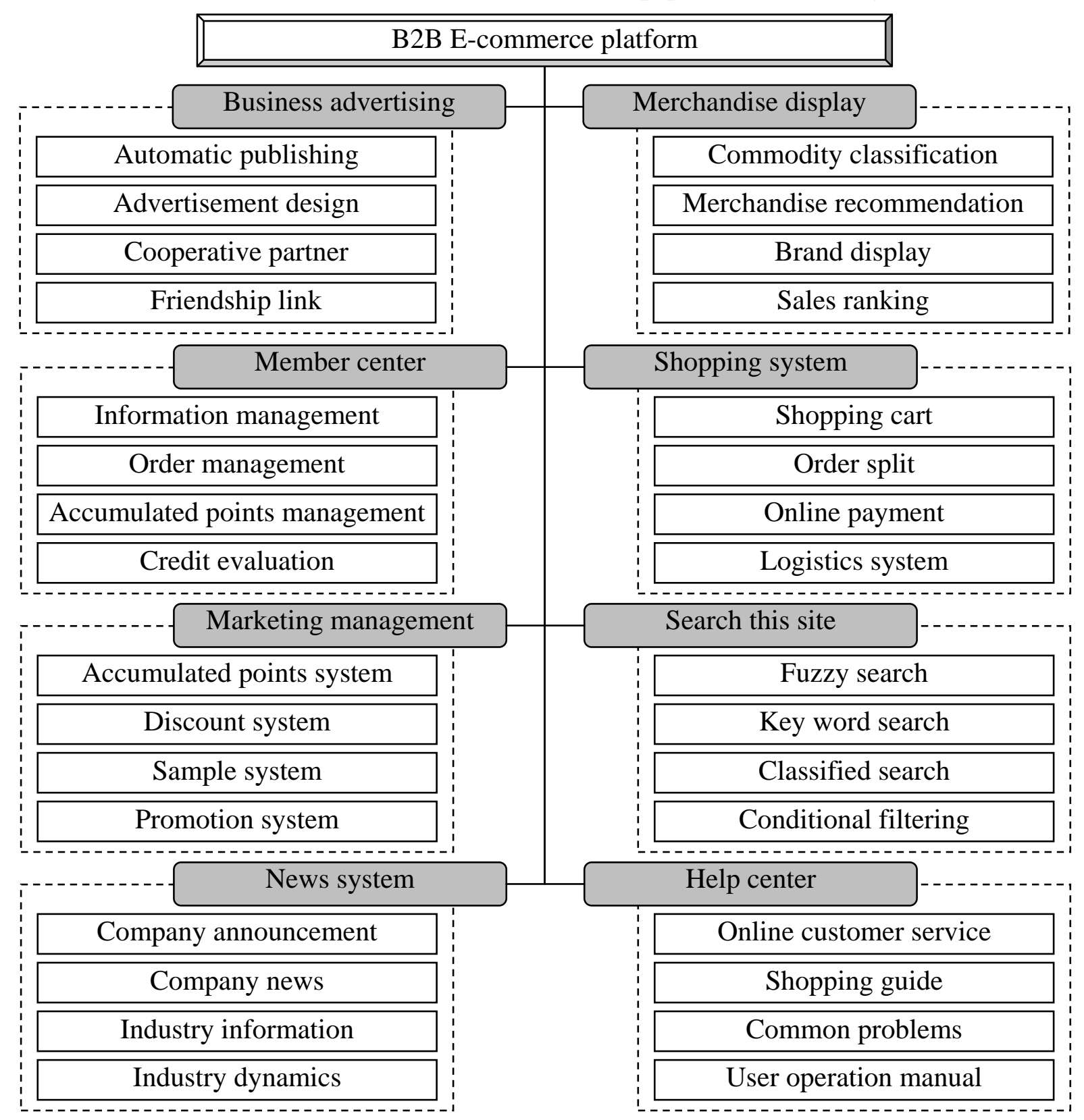

Fig. 2. Functional architecture on B2B E-commerce platform 


\section{Technology Framework Architecture}

The three layer architecture SSi by Struts, Spring and iBatis three technology framework combinations, from top to bottom, presentation layer, business logic layer and data persistence layer, cooperative interaction, to achieve the Web function and integration. The application of Sturts in control layer, responsible for handling user requests, control layer and business logic layer, data transfer, complete the user's request and response page display function; the application of Spring in the business logic layer, as a kind of based on the inversion of control (IoC) and aspect oriented programming (AOP) technology to realize the transaction manager. Bean and JDBC data source mechanism managed through dependency injection, complete the processing system of business logic and things and other functions; the application of iBatis in data persistence layer, through the establishment of a database table and the Java Bean between the semi automated object relation mapping, keep persistent data, complete the data exchange function. The technical framework architecture based on SSi is shown in Fig. 3.

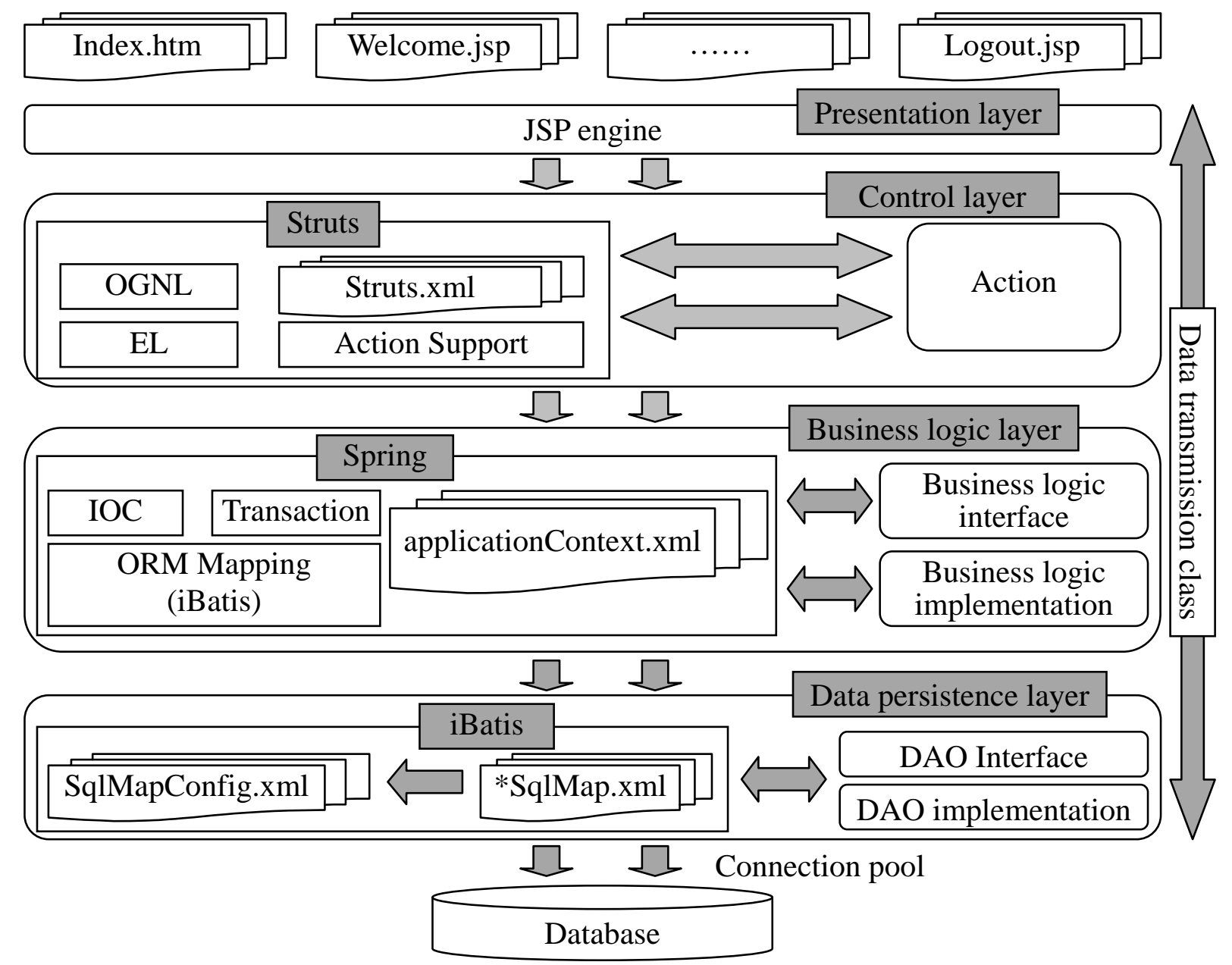

Fig. 3. Technology framework architecture based on SSi

(1) Presentation layer. Receive JSP page request, the corresponding class attribute action tags retrieved in the struts.xml configuration file, combined with the spring framework of inversion of control mechanism, gets an attribute value corresponding to the business logic interface from the spring container, specific business logic from the business logic layer, struts is responsible for processing results will return the corresponding JSP page display to the user. Developers need to configure struts.xml to write logical processing classes based on the business.

(2) Control layer. The Struts controller consists of the core controller and the service controller. The core controller is FilterDispatecher, which contains the control flow and processing mechanism within the framework. It is responsible for intercepting all user requests, and Filtet filters user requests when the user requests to arrive. The business controller Action and the business logic 
component need user implementation. While developing Action and business logic components, users also need to write relevant configuration files for use by the core controller FilterDispatcher.

(3) Business logic layer. In the way of "interface and implementation", Spring control is used to reverse the business logic implementation class provided by the container, and the transaction oriented logic is processed by using the aspect oriented programming technology to deal with the specific transaction. The object mapping technology fully supports the iBatis framework, and maintains the loose coupling between the entity class and the data table structure. The data interaction is achieved by transferring the data operation interface of the interface layer, and the result is returned to the presentation layer.

(4) Data persistence layer. The iBatis framework maps the input and output of the SQL statement, and solves the problem of database operation from the relation type to the object type, and gets the running result according to the persistent object. IBatis is "semi-automatic", need to write a SQL statement, the iBatis matches the Java object with the SQL statement, which not only plays a programmer to write SQL statements highly optimized, but also solves the mapping of Java objects and the input parameters and the result set.

\section{References}

[1] X. W. Zhou, " Comparison and innovation of business models of B2B e-commerce platform in China," Journal of Commercial Economics, vol. 33, no. 5, pp. 59-60, 2016.

[2] H. Tan, H. B. Zhang, "Design of B2B e-commerce platform for supply chain management," Manufacture Information Engineering of China, vol. 59, no. 7, pp. 23-26, 2012.

[3] Yao Cong, "What are the advantages of the B2B model?," http://www.xuexila.com/chuangye/hulianwang/375807.html, 2017-5-2.

[4] Instrument exhibition network, "B2B e-commerce process," http://www.18show.cn/show/165502/Affiche_258051.html, 2017-5-2.

[5] F. Y. Yao, "Construction of e-commerce integrated service platform based on B2B2C mode," Computer Programming Skills \& Maintenance, vol. 23, no. 10, pp. 9-17, 2016.

[6] L. Gao, "iBatis Persistence technology and its application in network teaching evaluation," Automation \& Instrumentation, vol. 36, no. 5, pp. 136-138, 2016.

[7] Script home, "SSI framework learning summary," http://www.jb51.net/article/55130.htm, 2017-5-2.

[8] L. J. Deng, Y. F. Zhang, J. M. Li, D. X. Dong, "Technology and application of SSI integration framework," Journal of Zhengzhou University of Light Industry(Natural Science Edition), vol. 30, no. 1, pp. 46-49, 2015. 